\title{
La brève histoire \\ du Centre de \\ recherche du CHU \\ Sainte-Justine
}

Emile Levy, Claude C. Roy

\section{Jogans \\ a taire grandir la vie.}

Au début du siècle dernier, plus d'un enfant sur quatre meurt avant l'âge d'un an. Irma Levasseur, première femme médecin canadienne-française, sensibilise son entourage à ce grave problème. Sa rencontre avec Justine Lacoste-Beaubien, ainsi que la générosité d'amis et de bienfaiteurs permettent l'ouverture du premier hôpital francophone pour enfants de Montréal, le 26 novembre 1907. L'hôpital est incorporé l'année suivante. C'est le premier jalon d'une longue histoire d'innovation.

Dès les premières années de notre institution aujourd'hui centenaire, la recherche a fait partie de sa mission. La fondatrice, madame Justine Lacoste-Beaubien, a voulu faire de l'hôpital Ste-Justine «non seulement un centre de guérison mais aussi un milieu scientifique d'études et de recherches... ». L'organisation d'un «laboratoire où nous pourrons contrôler nos travaux de clinique par des méthodes scientifiques des plus modernes et faire des travaux de recherches dont la cause infantile tout entière pourra bénéficier » est autorisée en 1914 sous l'impulsion du docteur Raoul Masson.

C'est en 1927 avec l'arrivée du docteur Pierre Masson, pathologiste de l'Université de Strasbourg, que le premier «virage scientifique » est effectué. L'année 1930 est marquée par le début de la publication des Annales Médico-chirurgicales de Ste-Justine, qui serviront de véhicule pour les travaux de recherche clinique. On organise, en 1940, un programme de conférenciers invités et, en 1945, le Conseil médical exige, par le biais d'une clause contractuelle, que chaque médecin résident se charge d'un travail scientifique. L'ère des spécialistes bien formés allait bientôt commencer grâce au programme subventionné par l'hôpital exigeant que les quatre années de formation soient couronnées par un stage d'un an à l'étranger.

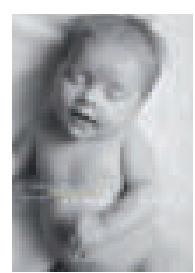

Fondation du Centre de recherche

C'est en 1960, après sa résidence en pédiatrie au Children's Hospital de Philadelphie et après sa formation en endocrinologie au Babies'Hospital

de I'Université Columbia à New York, que Jacques Ducharme a commencé sa carrière à Ste-Justine. Il fut le premier clinicien à faire de la recherche de laboratoire à une époque où elle n'avait pas encore droit de cité. Devenu directeur du département de pédiatrie en 1969 , il s'est fait le champion du recrutement de chercheurs détenteurs d'un PhD et de cliniciens chercheurs qui, comme lui, se reconnaissaient un rôle dans le domaine de la recherche de laboratoire. Conscient du besoin de regrouper les chercheurs dans des espaces appropriés, il fonda le Centre de recherche en 1974. Un an plus tard, le Centre comptait 32 chercheurs. Une quinzaine d'étudiants étaient inscrits aux études supérieures et les résultats des travaux faisaient l'objet de 104 articles et chapitres de livres. En 1977, il se voit confier le mandat de coordonner toutes les activités de recherche de l'hôpital, véritable point tournant pour l'instauration d'une culture scientifique au long cours.

En janvier 1978, le docteur Claude Roy lui succède. En cette Année de l'Enfant, promulguée par l'UNESCO, le directeur s'est efforcé de sensibiliser davantage les autorités locales et gouvernementales aux soucis de bénéficier de connaissances nouvelles. Effectivement, par une simple visite à la salle d'urgence et aux services hospitaliers, on se rendait vite compte que pour 


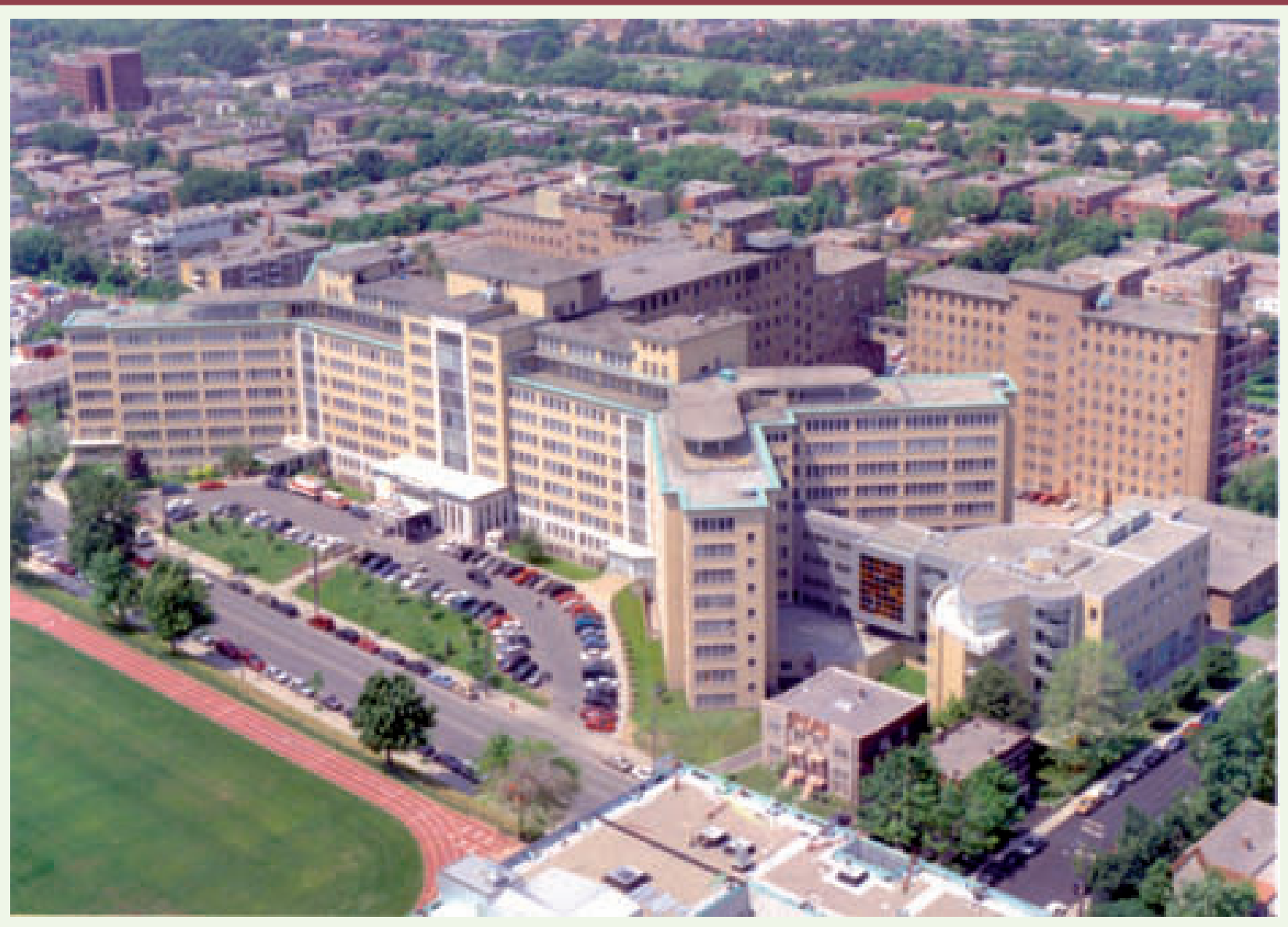

Vue aérienne de l'Hôpital Ste-Justine

une forte proportion de maladies requérant des soins secondaires et tertiaires, la médecine curative restait impuissante. Le docteur Roy voulait faire savoir que l'investissement de sommes considérables, pour améliorer l'accessibilité aux soins et offrir une meilleure qualité de soins, n'aurait de résultats qu'au prix d'efforts constants pour renouveler et mettre en commun des connaissances.

Le docteur Jacques Letarte prend la suite du docteur Roy, en décembre 1982. Le Centre maintenait le cap pour le développement et l'acquisition de nouvelles connaissances susceptibles d'être appliquées directement ou indirectement à la population pédiatrique. On vit alors le Centre renforcer ses ressources, tant en capital humain qu'en équipement, grâce à la détermination de ses mem-

bres, au soutien consenti par les organismes attitrés de financement de la recherche et, surtout, grâce à l'appui généreux de la population du Québec. En 1987, le docteur Charles-Hilaire Rivard assume la direction intérimaire du Centre de recherche. II faut

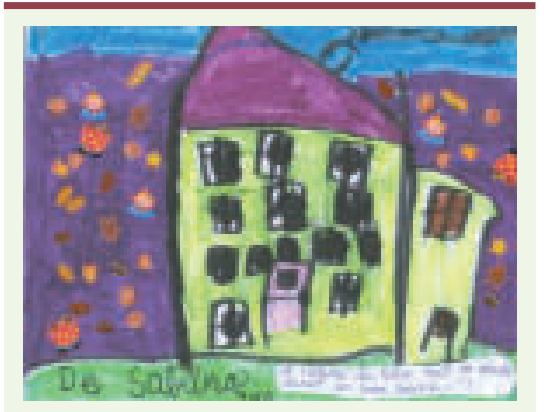

noter qu'au cours de ses 13 ans d'existence, le Centre s'était taillé une réputation fort enviable, comme en font foi le rayonnement de ses chercheurs, les 1718 publications dans des revues scientifiques de haut niveau et les 1905 communications de ses membres aux réunions de sociétés savantes nationales et internationales. Les travaux de recherche portaient déjà leurs fruits : nouvelles interventions thérapeutiques pour les enfants atteints de maladies chroniques, progrès en réadaptation physique et psychologique des handicapés, nouvelles approches pour améliorer la qualité de vie des jeunes patients porteurs de maladies chroniques.

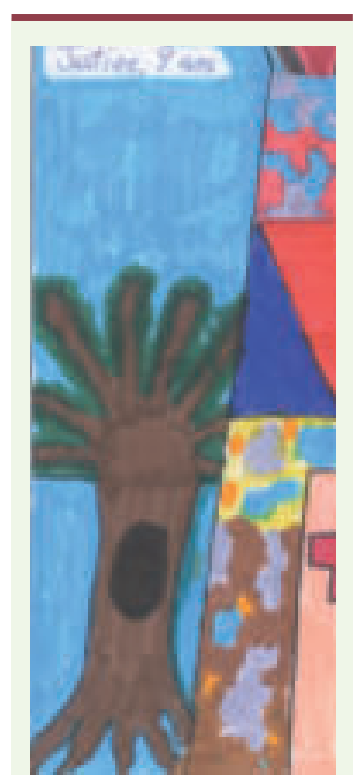




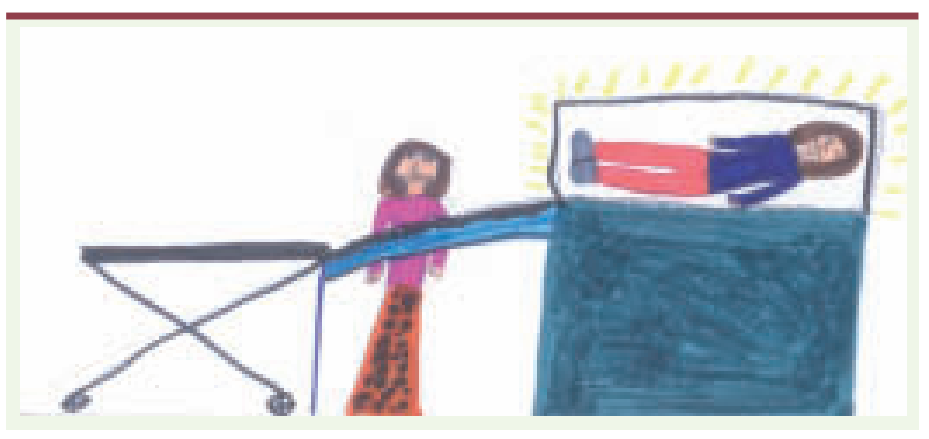

\section{L’ère de la valorisation de la recherche}

On assiste à la fin des années 1980 à une nette transformation des relations de la société avec la recherche qu'elle subventionne. Les chercheurs sont non seulement conviés à produire de nouvelles connaissances mais aussi à se responsabiliser pour leur transformation en nouveaux services et produits. Des mesures gouvernementales sont mises en place pour favoriser le grand $D$ du tandem $R \&$ D. Celles-ci conduisent à un rapprochement de la recherche universitaire avec le secteur privé, à une augmentation importante du capital de risque et à l'implantation de véritables incubateurs pour l'innovation aussi bien sociale que technologique. La création des Centres d'excellence par le gouvernement fédéral et des groupes de recherche du FRSQ témoigne d'un virage vers une recherche multidisciplinaire, multi-institutionnelle et multi-sectorielle axée sur le développement et la commercialisation. En 1988, le docteur Robert Collu prenait la direction du Centre. Durant les années de ses deux mandats, il a favorisé le recrutement de chercheurs qui sont venus se joindre aux équipes existantes ou qui ont ouvert de nouvelles disciplines. Par ailleurs, le docteur Collu a encouragé fortement l'essor de la génétique moléculaire et des sciences neurologiques. En outre, il a favorisé des progrès considérables en périnatalogie, hémato-oncologie, génétique, virologie, gastroentérologienutrition et orthopédie.

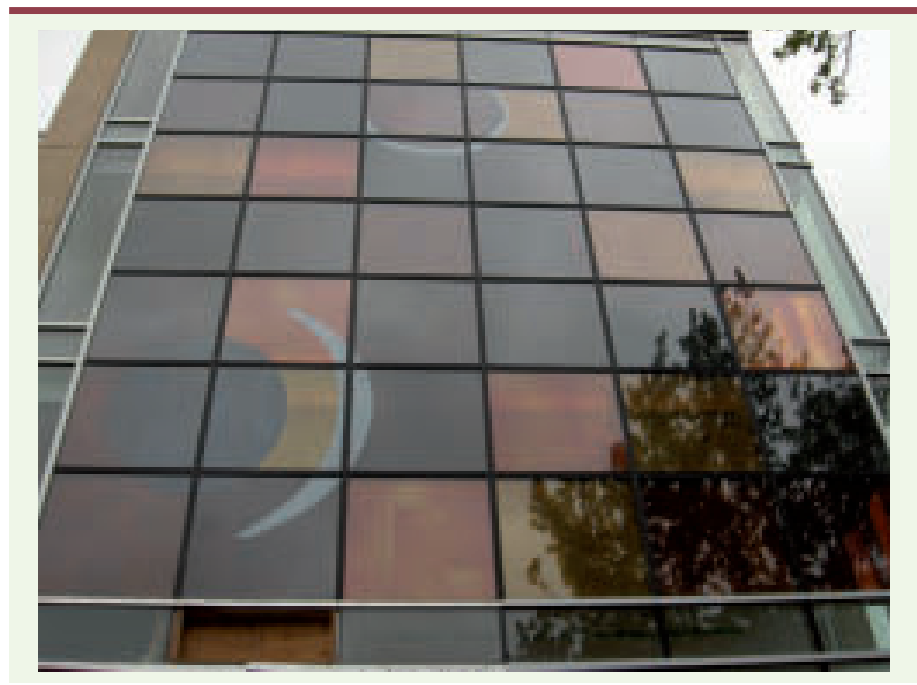

Verrière réalisée par l'artiste Marcelle Ferron - 1924-2001. Membre du groupe des automatistes.

\section{L'âge de la maturité}

En mai 1996, le docteur Emile Levy est nommé à la direction du Centre et il va le demeurer jusqu'en janvier 2006. II entendait non seulement continuer l'œuvre de ses prédécesseurs, mais aussi préparer activement le Centre de recherche à relever les défis du nouveau millénaire. On note l'apparition de regroupements des activités de recherche sous la forme d'axes ${ }^{1}$ qui ont beaucoup contribué aux progrès de l'entreprise de recherche, en privilégiant à l'intérieur de chaque axe, l'intégration de la recherche fondamentale, de la recherche clinique, de la recherche qui touche les programmes de soins et le système de santé, ainsi que celle qui étudie l'influence de la société et de l'environnement sur la santé des populations. Le modeste hôpital des débuts est maintenant devenu le CHU Ste-Justine, un centre d'excellence d'envergure mondiale en pédiatrie et en périnatalité. Sous la direction du DrEmile Levy, le Centre a pu tripler le nombre de chercheurs (ETP), augmenter de $60 \%$ le nombre des étudiants gradués et post-gradués, doubler le budget du Centre et des fonds de recherche, stimuler le transfert des connaissances (plus de 17 technologies ont été acheminées à l'industrie), développer les plates-formes technologiques et contribuer à la formation des réseaux.

\section{Innover et se développer}

En janvier 2006, le Dr Guy A. Rouleau, scientifique de renommée internationale, arrive à la tête du Centre de recherche. II a pour mandat d'optimiser la croissance du Centre au regard des événements à venir : la construction du nouveau centre, le recrutement stratégique de scientifiques novateurs et la collaboration étroite avec les partenaires institutionnels. $\varepsilon n$ effet, épaulé par l'Université de Montréal, la Fondation Ste-Justine et l'hôpital, il s'appuie sur les acquis du centre et il recherche les créneaux d'excellence pour exposer la présence du centre et de ses chercheurs dans la compétition internationale.

Le vibrant appel de nos jeunes patients et leur espoir de trouver chez nous des solutions à leurs problèmes de santé exigent créativité, courage et vision, ce dont l'hôpital SteJustine a toujours été doté. La lancée de Ste-Justine doit se poursuivre ${ }^{2} ! \diamond$

Brief history of the Ste-Justine Hospital Research Centre

${ }^{1}$ Anomalies du développement, facteurs de risque et prévention des psychopathologies; leucémie et cancer; maladies neuro-musculo-squelettiques; maladies métaboliques, génétiques et nutritionnelles; maladies virales et immunitaires; pathologies fœtomaternelles; Health outcomes

${ }^{2} \varepsilon n 2006$, le CHU Ste-Justine compte 450 lits, enregistre 19000 admissions annuellement et accueille 260000 patients en clinique externe provenant de l'ensemble du Québec. II est l'un des quatre plus importants centres pédiatriques en Amérique. Son Centre de recherche regroupe 163 chercheurs, 320 étudiants gradués et stagiaires postdoctoraux.

\section{TIRÉS À PART}

\section{ع. Levy}

